\title{
Differential Recruitment of Anterior Intraparietal Sulcus and Superior Parietal Lobule during Visually Guided Grasping Revealed by Electrical Neuroimaging
}

\author{
Eugene Tunik, ${ }^{1}$ Stephanie Ortigue, ${ }^{2}$ Serge V. Adamovich, ${ }^{3}$ and Scott T. Grafton ${ }^{2}$ \\ ${ }^{1}$ Department of Rehabilitation and Movement Science, University of Medicine and Dentistry of New Jersey, Newark, New Jersey $07107,{ }^{2}$ Department of \\ Psychology, 4D Brain Electrodynamics Laboratory, University of California, Santa Barbara, Santa Barbara, California 93106, and 32Department of Biomedical \\ Engineering, New Jersey Institute of Technology, Newark, New Jersey 07102-1982
}

\begin{abstract}
Dorsal parietal cortex is required for visually guided prehension. Transcranial magnetic stimulation to either the anterior intraparietal sulcus (aIPS) or superior parietal lobule (SPL) disrupts on-line adaptive adjustments of grasp when objects are perturbed. We used high-density electroencephalography during grasping to determine the relative timing of these two areas and to test whether the temporal contribution of each site would change when the task goal was perturbed. During object grasping with the right-hand, two distinct evoked responses were present over the 50-100 and 100-200 ms periods after movement onset. Distributed linear source estimation of these scalp potentials localized left lateralized sources, first in the aIPS and then the SPL. The duration of the response from the aIPS area was longer when there was an object perturbation. Initiation of a corrective movement coincided with activation in SPL. These data support a two-stage process: the integration of target goal and an emerging action plan within aIPS and subsequent on-line adjustments within SPL.
\end{abstract}

Key words: reaching; motor cognition; event-related potentials; human; brain microstates; brain plasticity

\section{Introduction}

Parietal cortex plays an essential role in sensorimotor integration. In this process, sensory and motor output signals are combined to provide an internal representation of the body state. This is needed for dynamic updating of action, i.e., for state estimation (Shadmehr and Krakauer, 2008). Evidence that parietal cortex is needed for updating can be found in patients with posterior parietal lesions (Wolpert et al., 1998; Gréa et al., 2002) and functional magnetic resonance imaging (fMRI) studies of grasping actions (Culham et al., 2003; Frey et al., 2005).

Sensorimotor integration can be reliably assayed by measuring compensatory motor responses in the face of unpredictable perturbations, forcing subjects to update an ongoing action (Desmurget and Grafton, 2000). Disruptive transcranial magnetic stimulation (TMS) to healthy subjects localizes the updating process both in time and space. In one study TMS to superior parietal lobule (SPL) and adjacent mid-intraparietal sulcus disrupted the reshaping of grip aperture when the target object changed size (Glover et al., 2005). In another target perturbation study, TMS administered at movement onset to anterior intraparietal sulcus (aIPS) impaired modulation of grip aperture when there was a

Received July 15, 2008; revised Nov. 4, 2008; accepted Nov. 6, 2008.

This work was supported by the Institute for Collaborative Biotechnologies Grant DAAD19-03-D-0004 from the U.S. Army Research Office and U.S. Public Health Service Grants NS44393 and NS33504.

Correspondence should be addressed to Dr. Scott T. Grafton, UCSB Brain Imaging Center, Department of Psychology, University of California, Santa Barbara, Santa Barbara, CA 93106. E-mail: grafton@psych.ucsb.edu. D0I:10.1523/JNEUROSCI.3303-08.2008

Copyright $\odot 2008$ Society for Neuroscience ～0270-6474/08/2813615-06\$15.00/0 change from a small to large object (Tunik et al., 2005). In a critical control experiment, subjects were required to adjust grip orientation rather than grip size. TMS to aIPS impaired reorienting the arm, suggesting that aIPS supports an internal representation of the task goal. Additional experiments showed that TMS to aIPS administered before movement onset had no effect (Rice et al., 2006). By manipulating the timing of TMS, the role of aIPS during updating was localized to a narrow temporal window of 50-75 ms after movement onset (Tunik et al., 2005). Given the temporal limitations of fMRI and the challenges of using single pulse TMS as a chronometric method the current experiment used electroencephalography (EEG) as an independent method to characterize cortical events occurring after movement onset.

On the basis of the above TMS studies it was hypothesized that aIPS is needed for the formation of an internal representation of a desired action (task goal). This contribution would occur early after movement onset and serve primarily to compare the desired and actual state. We tested this with high-density EEG as subjects reached to grasp an object (as in Tunik et al., 2005). Eventrelated-potentials (ERPs) were analyzed using a space-oriented brain electric field analysis to identify epochs of quasi-stable field configurations ("microstates") (Lehmann, 1987). The microstate localized to aIPS should appear shortly after movement onset. Second, we hypothesized that if aIPS is involved in representing a task goal then target perturbations should lead to prolonged recruitment in aIPS as part of the goal updating. Third, we hypothesized that the subsequent correction of the ongoing movement might recruit other parts of posterior parietal cortex, particularly 
SPL. We tested this by comparing the timing and sources of EEG events with kinematic markers that indicated when an adaptive response began (adjusted for sensorimotor delays).

\section{Materials and Methods}

\section{Participants}

Healthy (experiment 1: $n=12 ; 6$ males; mean age 23.4 \pm 3.9 ; experiment 2: $n=4 ; 2$ males; mean age $32.7 \pm 11.4$ ) subjects provided informed consent in accordance with the Human Subjects Committee of Dartmouth College. All were right handed by questionnaire (Oldfield, 1971). They had normal or corrected-to-normal visual acuity and no previous or current neurological or psychiatric impairment.

\section{Experiment 1}

Behavioral procedure. Participants sat at a comfortable reaching distance from a rectangular wood object. They were asked to reach toward the object and to grasp it with the right index finger and thumb such that the aperture was oriented along a vertical dimension relative to the floor (Tunik et al., 2005).

The target object was a $1 \times 1 \times 5 \mathrm{~cm}$ rectangle of wood mounted on the shaft of a motor (Kollmorgen model \#S6MH4) and positioned on a table along a sagittal axis from the subject's right shoulder (Tunik et al., 2005). Between trials, participants pressed a start button with the right index finger and awaited a $440 \mathrm{~Hz}, 400 \mathrm{~ms}$ tone that cued them to start a reach. The release of this button triggered rotation of the object (occurring in $50 \mathrm{~ms}$ ) resulting in a possible change in the size of the grasp dimension in the vertical axis from $1 \mathrm{~cm}$ to $5 \mathrm{~cm}$ (requiring subjects to readjust their finger aperture; PERTURBED condition). On the remaining trials, the graspable dimension was the same after rotation (UNPERTURBED condition. Each trial started with the object oriented horizontally so that the default grasp was with a small aperture. Subjects performed four blocks, 60 trials per block, with $25 \%$ of trials randomly assigned as PERTURBED trials.

Behavioral recording. Finger joint flexion-extension angles were measured with resistive bend sensors embedded in a glove (CyberGlove, Immersion). The glove contains individual 18 sensors, which acquire separate data for each major joint of the five fingers. The data glove (sampling rate, $100 \mathrm{~Hz}$ ) was connected to an RS-232 serial port of the National Instruments computer that also controlled the motor for rotating the object. Data from the sensors were converted from a voltage signal to an angular measurement in real time based on a calibration gain that was determined for each subject before the experiment. Calibration was performed for each subject by zeroing the flexion/extension sensors of the glove with the hand flat/fingers abducted, and assigning 90 degree values to the same sensors of the glove with the hand in a fist.

Behavioral data analysis and statistics. Because the pincer grasp was achieved primarily by changing the flexion-extension angle of the metacarpophalangeal (MCP) joint of the index finger, data analysis was limited only to this joint. Off-line, data were low-pass filtered $(10 \mathrm{~Hz})$ and analyzed using a custom-written Matlab (MathWorks) program. Movement onset was defined as the time at which the start button was released. Movement offset (the time when the rectangular object was grasped) was defined as the time at which the angular velocity of the MCP joint did not change by $>3 \%$ for at least $50 \mathrm{~ms}$. Five dependent measures were computed: (1) movement time (MT), the interval between movement onset and offset; (2) final MCP angle (MCPfinal), the MCP angle at movement offset; (3) peak MCP angle (MCPpeak), the maximal extension angle of the MCP joint; (4) time to MCPpeak (tMCPpeak), the interval between movement onset and the time of peak MCPe; and (5) latency of the adaptive response (latency), the interval of time between movement onset and the time when the mean MCP angle in the PERTURBED condition exceeded the mean $\pm 1 \mathrm{SD}$ of the MCP angle in the UNPERTURBED condition. This measure thus represents the time at which the adaptive (compensatory) response could be discerned at the kinematic level. We previously used this measure in similar perturbation-based paradigms to study online adaptive control mechanisms (Tunik et al., 2003, 2004, 2005; Rice et al., 2006). Dependent variables were subjected to one-way repeated-measures
ANOVA, with factor PERTURBATION (Levels: PERTURBED, UNPERTURBED). Statistical significance was set at $p<0.05$.

Electrophysiological recordings. Continuous electroencephalogram (EEG) was recorded from $128 \mathrm{AgCl}$ carbon-fiber coated electrodes using an Electric Geodesic Sensor Net (GSN300; Electrical Geodesic). During recordings, EEG was digitized at $500 \mathrm{~Hz}$, bandwidth at $0.01-200 \mathrm{~Hz}$, with the vertex electrode $(\mathrm{Cz})$ serving as an on-line recording reference; and impedances were kept $<50 \mathrm{k} \Omega$.

Electrophysiological image processing. Electrophysiological data were imported and analyzed in Cartool (version 3.33; Denis Brunet; http:// brainmapping.unige.ch/Cartool.htm). All trials were submitted to an automated threshold rejection criterion of $100 \mu \mathrm{V}$ and visually inspected for oculomotor (saccades, and blinks), muscles, and other artifacts. Surviving event-related potentials (ERPs) were computed covering $600 \mathrm{~ms}$ after the button release with a $500 \mathrm{~ms}$ prestimulus baseline. ERP data were then baseline corrected, and bandpass filtered between 1 and $30 \mathrm{~Hz}$. ERP data were then recalculated off-line against the average reference, and normalized to their mean global field power (Lehmann and Skrandies, 1980) before group averaging. The GFP, computed as the spatial SD of the scalp electric field, yields larger values for stronger electric fields and is calculated as the square root of the mean of the squared values recorded at each electrode (Lehmann and Skrandies, 1980; Murray et al., 2008). Channels with corrupted signals and channels showing substantial noise throughout the recording were interpolated to a standard 123channel electrode array using a three-dimensional spline procedure (Perrin et al., 1987). Then, two grand-mean ERPs were computed across the 12 subjects, one for each condition (i.e., condition 1: UNPERTURBED; condition 2: PERTURBED).

\section{Second-level electrophysiological data analysis}

Topographical analyses. ERP data were analyzed with space-oriented brain electric field analysis (Lehmann, 1987) using Cartool. This method has been described in detail previously (e.g., Ortigue et al., 2004; Murray et al., 2008). The space-oriented brain electric field approach is based on the empirical observation that the brain electric field configuration changes stepwise over time. Epochs of quasi-stable field configurations ("microstates") are concatenated by abrupt transitions in the brain electric field configurations. Variations in brain activity between experimental conditions produce differences in microstates. Accordingly, microstates index brief periods of quasi-stable spatial configurations of the active neural generators and thus are assumed to represent specific brain functions. To identify the start and end of each optimal microstate, a standard pattern analysis was performed using the grand-mean ERPs of each condition. This pattern analysis uses a hierarchical agglomerative cluster-algorithm to identify the predominant topographies (i.e., maps) and their sequence within a data set, implemented in Cartool (Tibshirani et al., 2005). The minimal number of maps that accounts for the greatest variance of the data set is determined based on a modified KrzanowskiLai criterion (Krzanowski and Lai, 1985). This pattern analysis is reference-free, and insensitive to amplitude modulation of the same scalp potential field across conditions. This pattern analysis was performed across time and experimental conditions to determine whether and when different experimental conditions engaged distinct configurations of intracranial generators. Then, the pattern of maps observed in the group-averaged data was statistically tested by comparing each of these maps with the moment-by-moment scalp topography of a subject's ERP from each condition. The optimal number of maps was fitted into the original data for each subject, using a competitive fitting procedure (Brandeis et al., 1995). This "fitting" procedure determines whether a given experimental condition is more often described by one map versus another at the individual level. The amount of time every given stable configuration existed was tested by repeated-measures ANOVA, significant at $p<0.05$.

Distributed intracranial source estimations (LAURA). The above topographic pattern analysis defined time periods to estimate intracranial sources using the Local Auto-Regressive Average (LAURA) (Grave de Peralta Menendez et al., 2001). LAURA inverse solution was estimated in a realistic $3 \mathrm{D}$ solution space for every condition using a lead field calculated on a realistic average brain model provided by the Montreal Neu- 


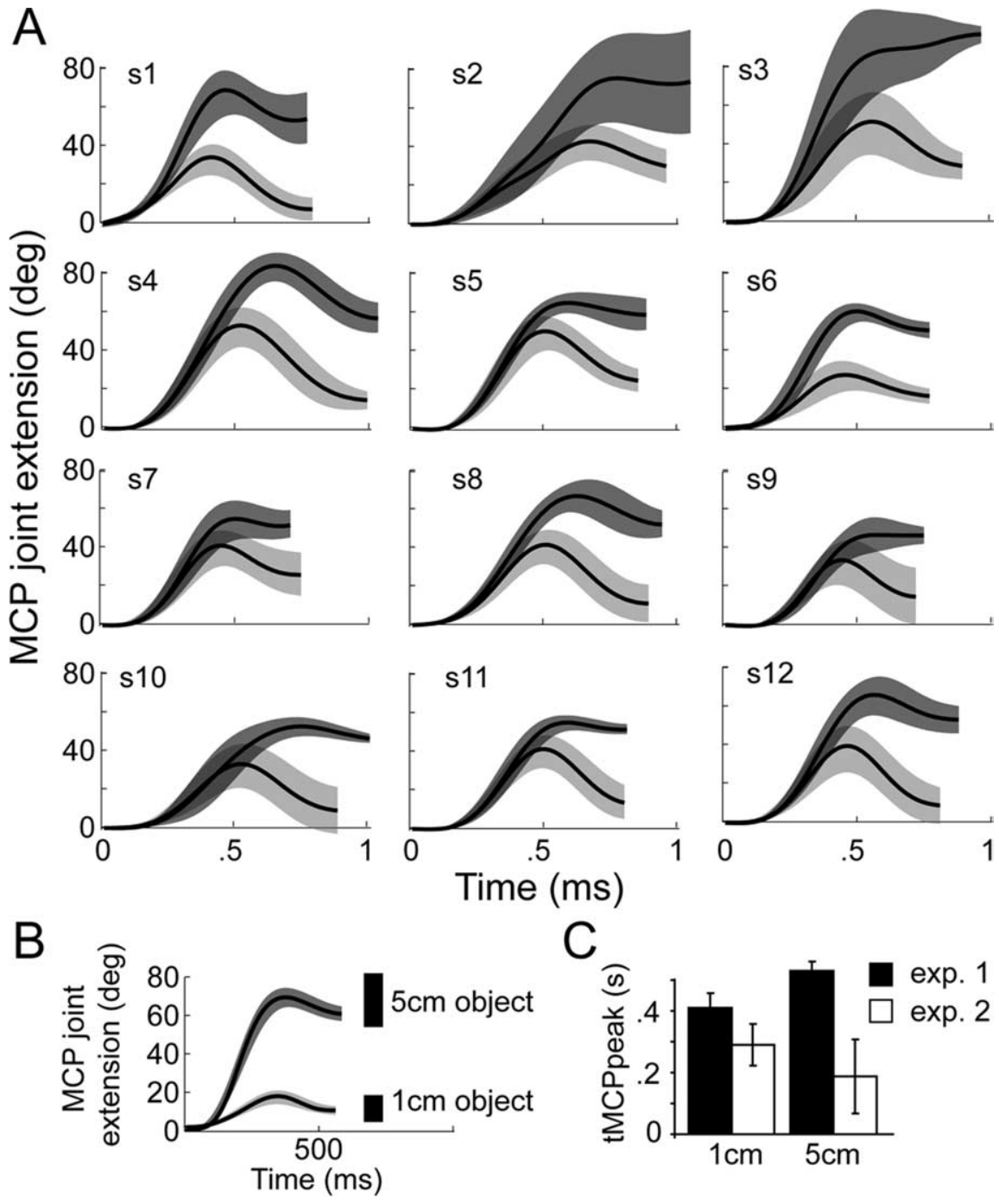

Figure 1. $A$, Mean MCP angle $\pm 1 S D$ (shaded regions) ( $y$-axis) profiles for 12 subjects in the perturbation experiment (experiment 1). The perturbation occurred from 1 to $5 \mathrm{~cm}$. Light gray, $1 \mathrm{~cm}$ object; dark gray, $5 \mathrm{~cm}$ object. $\boldsymbol{B}$, The mean MCP angle \pm 1 SD plotted over time for a representative subject in experiment 2 (no perturbation). $C$, The mean \pm 1 SD time to peak $M C P$ angle in the perturbation (experiment 1) and no-perturbation (experiment 2) experiments.

rological Institute (MNI) with the software "SMAC" (Spinelli et al., 2000). The head model included 3005 solution points, selected from a $6 \times 6 \times 6 \mathrm{~mm}$ grid equally distributed within the gray matter. Source estimations were rendered on an average standard brain supplied by Cartool. Transformation between the MNI coordinate system and that of Talairach and Tournoux was performed with Cartool.

\section{Experiment 2}

Experiment 2 characterized the kinematic profiles for grasping the $1 \mathrm{~cm}$ and $5 \mathrm{~cm}$ object under unperturbed conditions. The setup was identical to experiment 1 except that only glove data were collected and grasp conditions were blocked ( 10 trials per object size). Kinematics were compared between experiment 1 and 2 using repeated-measures ANOVAs (within-group factor: OBJECT SIZE [small, large]; between-group factor: EXPERIMENT [exp1, exp2]).

\section{Results}

\section{Motor performance}

Experiment 1

Subjects used a pincer grip to grasp the target object, whose size remained UNPERTURBED (narrow) on a majority of trials and was unexpectedly increased on $25 \%$ of random trials. Subjects compensated for this perturbation by significantly increasing the
MCPfinal in the PERTURBED $\left(73.6^{\circ}\right.$ $\left.\pm 5.9^{\circ}\right)$ relative to the UNPERTURBED $\left(16.2^{\circ} \pm 5.8^{\circ}\right)$ trials (paired $t$ test: $t_{11}=$ 38.5, $p<0.001$ ) (Fig. 1A). The MCPpeak also significantly increased in the PERTURBED $\left(94.5^{\circ} \pm 9.4^{\circ}\right)$ relative to the UNPERTURBED $\left(47.2^{\circ} \pm 7.5^{\circ}\right)$ condition $\left(t_{11}=20.1, p<0.001\right)$, a scaling effect that is consistent with the stereotypical scaling of peak aperture that occurs during pincer grasping. Accordingly, the time to MCPpeak (tMCPpeak) occurred significantly later in PERTURBED (529 $\pm 30.8 \mathrm{~ms})$ relative to UNPERTURBED $(410 \pm 46.7 \mathrm{~ms})$ trials $\left(t_{11}=8.6, p<0.001\right)$, although this did not have as pronounced an impact on MT, which was only marginally significant between the two conditions (PERTURBED condition: $764.2 \pm 45.1 \mathrm{~ms}$; UNPERTURBED condition: $726.7 \pm 78.1$ ms; paired $t$ test: $\left.t_{11}=2.26, p=0.049\right)$. Finally, we quantified the latency of the onset of the adaptive response by identifying the time at which the MCP angle in the PERTURBED trials exceeded the mean + 1SD of the mean MCP angle in the UNPERTURBED trials. The group mean (SD) latency of the adaptive response was 271.3 $\mathrm{ms}( \pm 95.2 \mathrm{~ms})$ from the time of the perturbation (which occurred at movement onset), consistent with latencies of adaptive responses reported in other similar paradigms (i.e., Rice et al., 2006).

\section{Experiment 2}

The purpose of experiment 2 was to obtain additional kinematic evidence from unperturbed trials to show 1) the adaptation of experiment 1 was an active response occurring in the early part of movement and not present with unperturbed grasp to large objects; and 2) the latency for discriminating between a grasp to a large versus small object under unperturbed conditions. This latency could be compared against available data of electromechanical delay and central conduction time to verify the onset of the updating response in experiment 1.

Figure $1 B$ shows the mean $( \pm 1 \mathrm{SD}) \mathrm{MCP}$ angle trajectory profiles of one representative subject grasping the small and large object under unperturbed conditions. The MCPpeak in experiment 2 was $88.8^{\circ} \pm 4.7^{\circ}$ and $41.3^{\circ} \pm 7.6^{\circ}$ for the large and small objects, respectively. The MCPfinal was $74.1^{\circ} \pm 9.9^{\circ}$ and $13.8 \pm$ $5^{\circ}$ for the large and small objects, respectively (EXPERIMENT main effect/interactions, $p>0.2$ ) suggesting that the perturbation did not beckon changes in joint excursion magnitude. However, tMCPpeak revealed a significant EXPERIMENT main effect (small: $290 \pm 67 \mathrm{~ms}$; large: $188 \pm 119 \mathrm{~ms})\left(F_{(1,14)}=86.2 ; p<\right.$ $0.0001)$ and EXPERIMENT $\times$ OBJECT SIZE interaction $\left(F_{(1,14)}\right.$ $=26.8 ; p=0.0001)$. Further analysis revealed that the interaction effect was attributed to an increase in the tMCPpeak in experiment 1 when adapting to the perturbation, an effect that did not occur for unperturbed grasp (experiment 2) (Fig. 1C). For MT, only a main effect of EXPERIMENT (large: $594 \pm 92$ ms; small: 
$577 \pm 43 \mathrm{~ms})$ was noted $\left(F_{(1,14)}=24.3\right.$; $p<0.0002)$, which is expected given that no extra time was needed in the unperturbed experiment to reprogram a compensatory movement. Finally, the latency at which we could detect a reliable difference in MCP angle between the $1 \mathrm{~cm}$ and 5 $\mathrm{cm}$ grasps was $72.5 \pm 72 \mathrm{~ms}$. These data demonstrate that reliable differences in the MCP angle could be detected very early when planning the two different grasps under unperturbed conditions. Conversely, the latency to reprogram the grasp in response to a perturbation (see experiment 1) occurred at $271.3 \mathrm{~ms}$, or $198.8 \mathrm{~ms}$ later $\left(t_{13}=3.6, p=0.003\right)$.

\section{Electrophysiological results}

The topographic pattern analysis identified six time periods of stable topography across the collective $600 \mathrm{~ms}$ postbutton release period for both of the conditions (UNPERTURBED and PERTURBED). The epochs with stable topographies were: UNPERTURBED condition: microstate 1 : 0-50 ms; 2: 50-100 ms; 3: 100-200 ms; 4: 200-354 ms; 5: 354-530 ms; 6: 530-600 ms; PERTURBED condition: microstate 1 : 0-50 ms; 2: 50-150 ms; 3: 150-206 ms; 4: 206-348 ms; 5: 348-536 ms; 6: 536-600 ms (Fig. 2).

The beginning of both conditions (i.e., microstate 1: 0-50 ms) was characterized by similar stability of the intracranial generator configurations. The next two microstates (2 and 3, 50-200 ms after movement onset) showed significantly different patterns of stability between the UNPERTURBED and PERTURBED conditions (Fig. 2). Microstate 2 was present for a longer amount of time (i.e., from 50 to $150 \mathrm{~ms}$ ) for the PERTURBED than for the UNPERTURBED condition (from 50 to $100 \mathrm{~ms}$ ) (Figs. 2, 3). Critically, microstate 2 was present for a longer duration in the PERTURBED condition, whereas microstate 3 was present for a longer duration in the UNPERTURBED condition $\left(F_{(1,11)}=8.67 ; p=0.013\right)$ (Fig. 3). A distributed linear source estimation of microstate 2 (for both PERTURBED and UNPERTURBED conditions) revealed a leftlateralized current density source in the intraparietal sulcus with the maximum localized to the anterior extent of the sulcus [local maximum: $-34,-56,48 \mathrm{~mm}(x, y, z$ Talairach coordinates $)$ ] (Fig. 3). However, microstate 3 was characterized by sources within the superior parietal lobule (local maximum: $-16,-51$, $62 \mathrm{~mm}$ ) (Fig. 3).

Source estimations across the remaining microstates revealed a sequence of likely generators mainly within the left parietal lobe, summarized in Figure 4. The earliest source estimation (microstate 1) was localized mainly to the left lateral temporooccipital cortex (local maximum: $-3,-82,1 \mathrm{~mm}$ ). Microstate 4 was characterized by activation in the parieto-occipital sulcus (local maximum: $-15,-78,35 \mathrm{~mm}$ ) and also a moderate activation in the frontal gyrus $(-31,-2,52 \mathrm{~mm})$, and occipitotemporal regions (with a maximum in the inferior temporal gyrus: $-49,-67,6 \mathrm{~mm}$ ). Microstate 5 was characterized by a bilateral activation in the parieto-occipital sulcus (local maximum: $-15,-78,35 \mathrm{~mm}$ ). Finally, the source estimation of microstate 6 revealed bilateral occipital as well as anterior cingulate (Brodmann's Area 32) activity (local maximum: -3, 35, $-10 \mathrm{~mm})$.

\section{Discussion}

The current study is the first in humans to relate the timing of cortical activity through high-density electrical mapping to movement kinematics in a prehension task. EEG has been used previously to characterize temporally specific potentials over parietal cortex during sensorimotor transformation (Waszak et al., 2005) but has not been related directly to motor updating or control. Our behavioral results showed that for perturbed trials, the latency of a compensatory response was reliably detected an average of $271 \mathrm{~ms}$ after the target perturbation. This serves as a marker for identifying the critical microstate for initiating the on-line updating of an ongoing movement. Analysis of tMCPpeak and MT between experiments 1 and 2 confirmed that the adaptive response was initiated in the early portion of the movement. Subtracting the amount of time attributed to the electromechanical delay (published mean $64 \mathrm{~ms}$, range 45-82 ms) (Cavanagh et al., 1979; Onishi et al., 2006) and central-peripheral conduction time (21 ms) (Rayegani et al., 2008) yields an upper bound of $186 \mathrm{~ms}$, i.e., the approximate amount of time required to perceive a target perturbation, reprogram and then initiate an 


\section{Microstate 2}

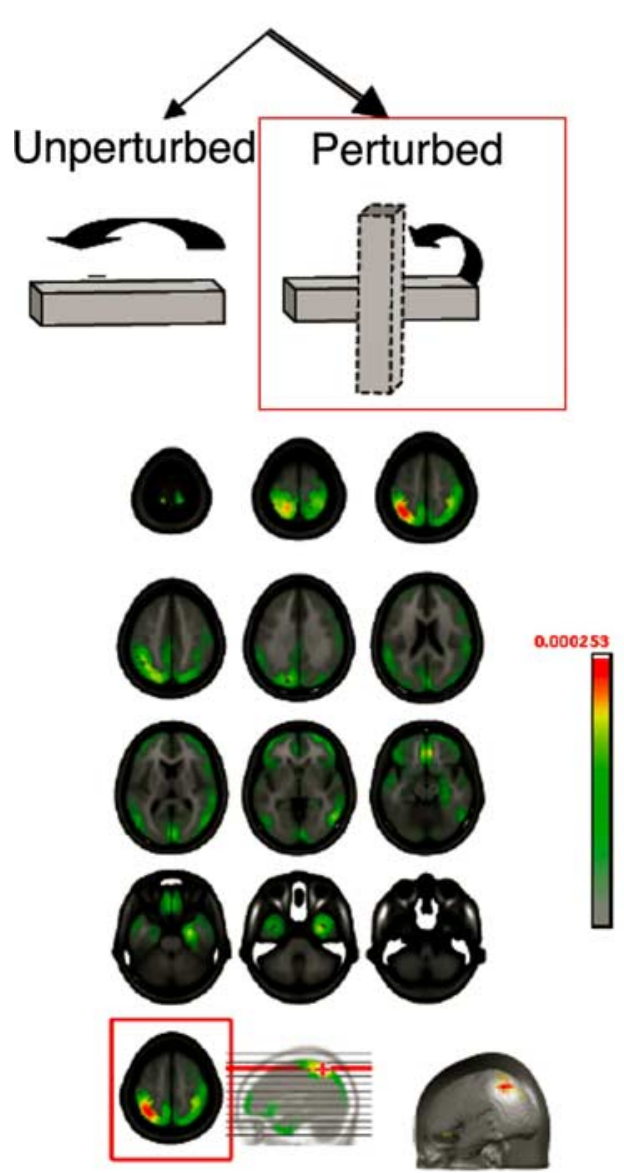

Microstate 3

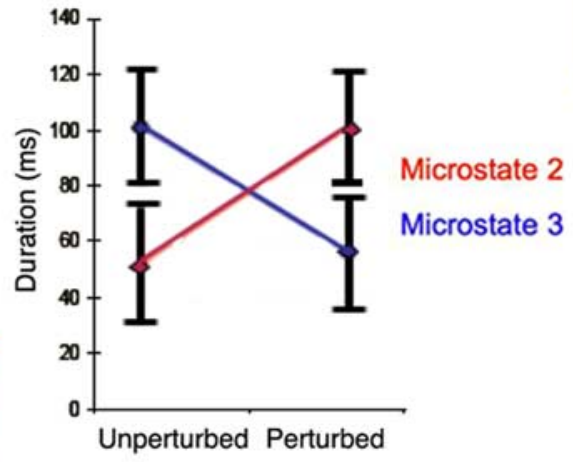

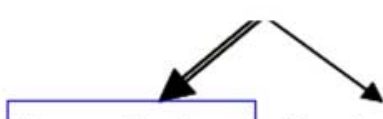

Unperturbed Perturbed
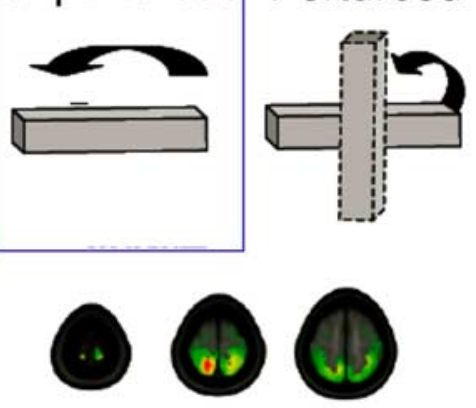

O

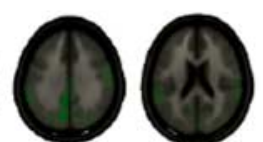

0.000397
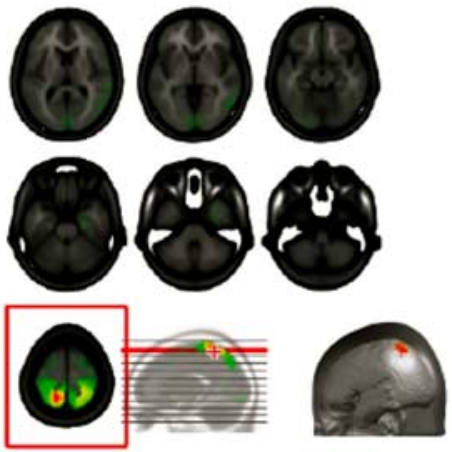

Figure 3. Electrical neuroimaging results for the two microstates in the $50-206 \mathrm{~ms}$ time window. Left, Source localization of microstate 2 revealed a left-lateralized current density source in the intraparietal sulcus with the maximum (bottom row) in the anterior extent of the sulcus. Right, Source localization of microstate 3 revealed a left-lateralized current density source with a maximum in the superior parietal lobule (bottom row). Middle, Plot of the duration of each microstate as a function of condition. Microstate 2 was significantly longer in the PERTURBED case whereas microstate 3 was significantly longer for the UNPERTURBED case $\left(F_{(1,31)}=8.67, p<0.013\right)$.

online response. Note that this "reprogramming" delay is remarkably similar to the difference in the latencies between experiment 1 and 2 (198.8 ms), further validating our observation that reprogramming occurred in this timeframe. The reprogramming delay of $186 \mathrm{~ms}$ fits within microstate 3 defined by EEG, whose activation was noted between 100 and $200 \mathrm{~ms}$ after the perturbation and localized over the left superior parietal lobule. This timing supports our hypothesis that SPL plays a direct role in the initiation of on-line updating for early movement corrections.

Our EEG data demonstrates that microstate 2, occurring between 50 and $100 \mathrm{~ms}$ post movement onset localizes to aIPS. The timing of microstate 2 fits well with experiments demonstrating a particular sensitivity of aIPS to TMS on task performance when it is applied near movement onset, whether a perturbation is delivered or not (Tunik et al., 2005; Rice et al., 2006). The findings argue that aIPS is important for integrating target information with initial motor commands.

These results show a significant recruitment within aIPS occurring before SPL. Furthermore, they show longer recruitment of microstate 2 (aIPS) than 3 (SPL) when there is perturbation of a target. These observations support a two-stage model, in which aIPS is involved in the initial state estimation as an efference copy becomes available. With sufficient error between the desired and actual state there is additional recruitment of this area to initiate corrections that are mediated in part by the SPL.

From a methodological perspective, the spatial and temporal consistency between the current high density electrical mapping and previous TMS data using the same task suggests that the EEG could be used to effectively identify spatiotemporal windows of functional vulnerability in the cortex. During these windows, cortex should be particularly sensitive to single pulse TMS. This could greatly enhance the efficiency in finding sensitive periods of cortical processing in chronometric TMS experiments.

\section{References}

Brandeis D, Lehmann D, Michel CM, Mingrone W (1995) Mapping eventrelated brain potential microstates to sentence endings. Brain Topogr 8:145-159.

Cavanagh PR, Komi PV (1979) Electromechanical delay in human skeletal muscle under concentric and eccentric contractions. Eur J Appl Physiol Occup Physiol 42:159-163.

Culham JC, Danckert SL, DeSouza JF, Gati JS, Menon RS, Goodale MA (2003) Visually guided grasping produces fMRI activation in dorsal but not ventral stream brain areas. Exp Brain Res 153:180-189.

Desmurget M, Grafton S (2000) Forward modeling allows feedback control for fast reaching movements. Trends Cogn Sci 4:423-431.

Frey SH, Vinton D, Norlund R, Grafton ST (2005) Cortical topography of 


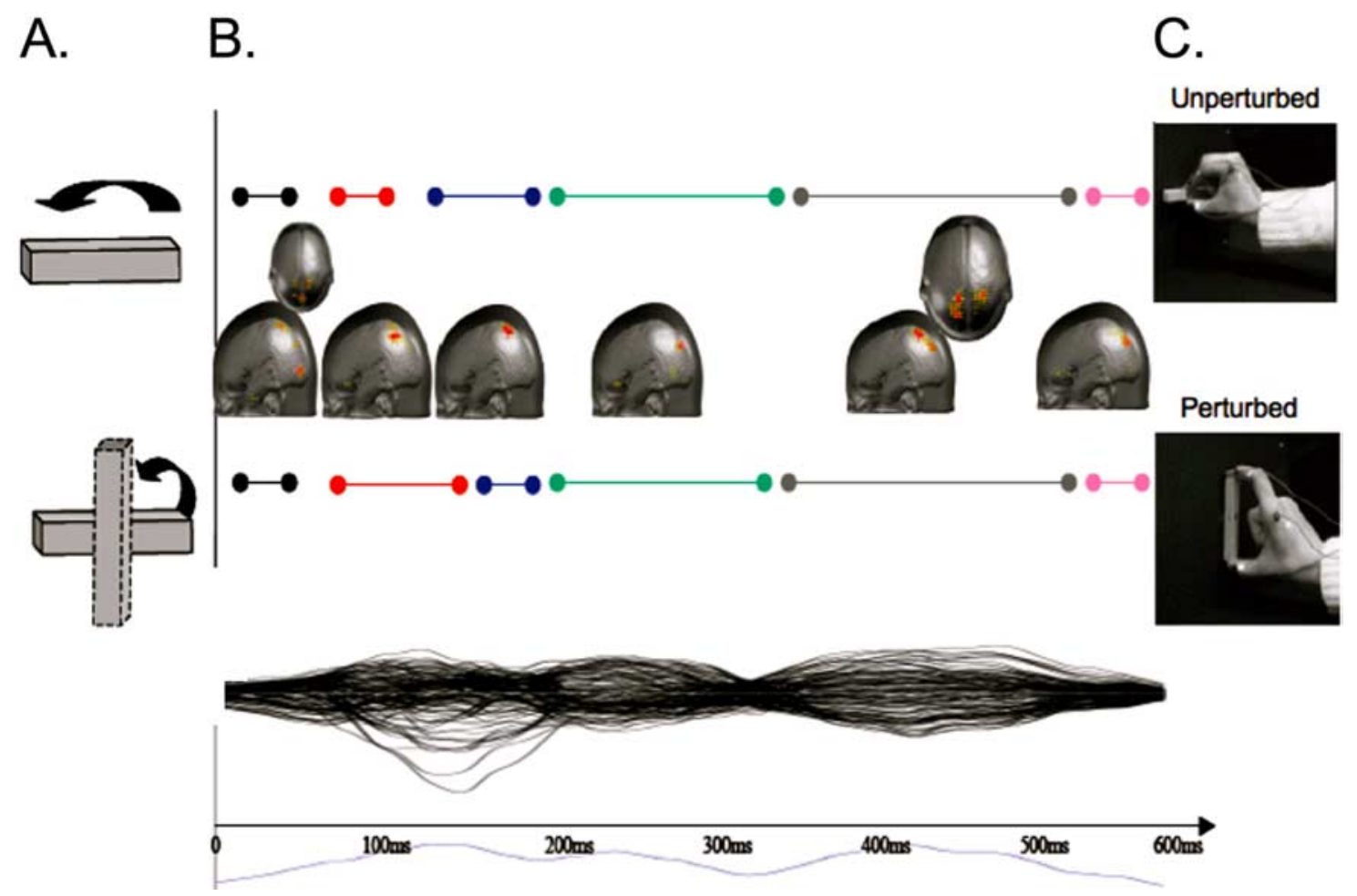

Figure 4. Distributed linear source estimation over the entire trial. $\boldsymbol{A}$, Target position during reach. $\boldsymbol{B}$, Maximal source estimations for UNPERTURBED (top) and PERTURBED (bottom) conditions are projected onto the brain surface. The duration of the six microstates are identified by the colored bars. C, Movement outcome for each condition. Lower plot shows individual potentials for each electrode and the global field power over all electrodes (in blue below the $x$-axis).

human anterior intraparietal cortex active during visually guided grasping. Brain Res Cogn Brain Res 23:397-405.

Glover S, Miall RC, Rushworth MF (2005) Parietal rTMS disrupts the initiation but not the execution of on-line adjustments to a perturbation of object size. J Cogn Neurosci 17:124-136.

Grave de Peralta Menendez R, Gonzalez Andino S, Lantz G, Michel CM, Landis T (2001) Noninvasive localization of electromagnetic epileptic activity. I. Method descriptions and simulations. Brain Topogr 14:131-137.

Gréa H, Pisella L, Rossetti Y, Desmurget M, Tilikete C, Grafton S, Prablanc C, Vighetto A (2002) A lesion of the posterior parietal cortex disrupts on-line adjustments during aiming movements. Neuropsychologia 40:2471-2480.

Krzanowski W, Lai YT (1985) A criterion for determining the number of groups in a data set using sum of squares clustering. Biometrics 44:23-34.

Lehmann D (1987) Principles of spatial analysis. In: Handbook of electroencephalography and clinical neurophysiology, vol 1: Methods of analysis of brain electrical and magnetic signals (Gevins AS, Remond A, eds), pp 309-354. Amsterdam: Elsevier.

Lehmann D, Skrandies W (1980) Reference-free identification of components of checkerboard-evoked multichannel potential fields. Electroencephalogr Clin Neurophysiol 48:609-621.

Murray MM, Brunet D, Michel CM (2008) Topographic ERP analyses: a step-by-step tutorial review. Brain Topogr 20:249-264.

Oldfield RC (1971) The assessment and analysis of handedness: the Edinburgh inventory. Neuropsychologia 9:97-113.

Onishi H, Soma T, Kameyama S, Oishi M, Fuijmoto A, Oyama M, Furusawa AA, Kurokawa Y (2006) Cortical neuromagnetic activation accompanying two types of voluntary finger extension. Brain Res 1123:112-118.

Ortigue S, Michel CM, Murray MM, Mohr C, Carbonnel S, Landis T (2004) Electrical neuroimaging reveals early generator modulation to emotional words. Neuroimage 21:1242-1251.

Perrin F, Pernier J, Bertrand O, Giard MH, Echallier JF (1987) Mapping of scalp potentials by surface spline interpolation. Electroencephalogr Clin Neurophysiol 66:75-81.

Rayegani SM, Hollisaz MT, Hafezi R, Nassirzadeh S (2008) Application of magnetic motor stimulation for measuring conduction time across the lower part of the brachial plexus. J Brachial Plex Peripher Nerve Inj 3:7.

Rice NJ, Tunik E, Grafton ST (2006) The anterior intraparietal sulcus mediates grasp execution, independent of requirement to update: new insights from transcranial magnetic stimulation. J Neurosci 26:8176-8182.

Shadmehr R, Krakauer JW (2008) A computational neuroanatomy for motor control. Exp Brain Res 185:359-381.

Spinelli L, Andino SG, Lantz G, Seeck M, Michel CM (2000) Electromagnetic inverse solutions in anatomically constrained spherical head models. Brain topography 13:115-125.

Tibshirani R, Walther G, Botstein D, Brown P (2005) Cluster validation by prediction strength. J Comput Graphical Stat 14:511-528.

Tunik E, Poizner H, Levin MF, Adamovich SV, Messier J, Lamarre Y, Feldman AG (2003) Arm-trunk coordination in the absence of proprioception. Exp Brain Res 153:343-355.

Tunik E, Adamovich SV, Poizner H, Feldman AG (2004) Deficits in rapid adjustments of movements according to task constraints in Parkinson's disease. Mov Disord 19:897-906.

Tunik E, Frey SH, Grafton ST (2005) Virtual lesions of the anterior intraparietal area disrupt goal-dependent on-line adjustments of grasp. Nat Neurosci 8:505-511.

Waszak F, Wascher E, Keller P, Koch I, Aschersleben G, Rosenbaum DA, Prinz W (2005) Intention-based and stimulus-based mechanisms in action selection. Exp Brain Res 162:346-356.

Wolpert DM, Goodbody SJ, Husain M (1998) Maintaining internal representations: the role of the human superior parietal lobe. Nat Neurosci 1:529-533. 